\title{
Impacts des changements de politique dans un contexte de crise mondialisée sur les acteurs des filières cotonnières d'Afrique centrale
}

\author{
Politique et crise cotonnières en Afrique centrale
}

\author{
Emmanuel Mbétid-Bessane ${ }^{1}$ \\ Koye Djondang ${ }^{2}$ \\ Michel Havard ${ }^{3}$ \\ David Kadékoy-Tigagué ${ }^{4}$ \\ ${ }^{1}$ Université de Bangui \\ BP 1983 Bangui \\ Centrafrique \\ <mbetid@hotmail.com> \\ ${ }^{2}$ Institut tchadien de recherche agronomique \\ (ltrad), \\ Pôle régional de recherche appliquée \\ au développement des systèmes agricoles \\ d'Afrique Centrale (Prasac) \\ BP 5400 \\ N'Djaména \\ Tchad \\ <djondang_koye@yahoo.fr> \\ ${ }^{3}$ Centre de coopération internationale en \\ recherche agronomique pour le \\ développement (Cirad) \\ UMR Innovation \\ 73, rue J-F. Breton \\ 34398 Montpellier cedex 5 \\ France \\ <michel.havard@cirad.fr> \\ ${ }^{4}$ Institut centrafricain de recherche agricole \\ (ICRA), \\ Pôle régional de recherche appliquée \\ au développement des systèmes agricoles \\ d'Afrique Centrale \\ BP 122 \\ Bangui \\ Centrafrique \\ <kadekoy@yahoo.fr>
}

Tirés à part : M. Havard

\begin{abstract}
Résumé
La réorganisation des filières cotonnières en réponse à la crise du commerce mondial a des effets sur les économies nationales, les revenus des producteurs et le développement des zones de savanes d'Afrique centrale. Cet article nourrit la réflexion sur ces questions en analysant les déterminants et les effets de la réorganisation, mais aussi les réactions des producteurs à ces changements. À cette fin, les travaux de recherche sur une dizaine d'années du Pôle régional de recherche appliquée au développement des systèmes agricoles d'Afrique centrale sur les filières cotonnières et les exploitations agricoles sont mobilisés. La réorganisation des filières amène les opérateurs industriels à se focaliser sur la commercialisation et la transformation du coton, abandonnant progressivement les activités de service et de développement, dont certaines sont reprises par les organisations de producteurs. Avec la crise, les opérateurs recherchent une meilleure rentabilité de leurs activités cotonnières et de leurs infrastructures, et les producteurs, voyant leurs revenus baisser, développent des stratégies de recomposition de leurs activités, où la place du coton diminue. Le devenir des opérateurs, de leurs infrastructures et des exploitations agricoles est mis en question. Dans cette période d'incertitude, les acteurs de ces filières doivent être accompagnés pour s'adapter aux changements et maintenir leurs revenus, et les mécanismes d'appui au développement de ces zones de savanes demandent à être redéfinis.
\end{abstract}

Mots clés : Afrique centrale ; agriculteurs ; coton ; crise économique ; politique agricole.

Thèmes : économie et développement rural ; productions végétales ; systèmes agraires.

\section{Abstract \\ Impacts of policy change on cotton sector stakeholders in Central Africa in the context of the global crisis}

The cotton crisis and reorganisation of the production chains affect the national economy and producers' revenue, thus raising the issue of development prospects in the Central African savannahs. This paper addresses the mentioned phenomena by analyzing factors and implications of the production chain reorganization and the cotton crisis as well as farmers' reactions to the evolving contexts. The paper is based on the outcome of about ten years' research studies related to cotton production chains and farming systems carried out by the Regional Pole of Applied Research for the Development of Farming Systems in Central Africa. The restructuring process of the production chains has led cotton companies to concentrate their activities on production and processing of cottonseeds, progressively abandoning other activities related more directly to service supply for development, some of which have been taken over by producers' organisations. The cotton crisis has pushed cotton companies to become more concerned with profitability and better use of their infrastructures. On the producers' side, the cotton crisis has resulted in reduced income to which they reacted by diminishing the area devoted to cotton production. The future of societies based on cotton production, their infrastructures and even cotton farming is debated. Under great uncertainty, cotton 
sector stakeholders require accompaniment so as to maintain their revenue following the redefinition of support mechanisms affecting the development of the savannahs in Central Africa.

Key words: agricultural policies; Central Africa; cotton; economic crisis; farmers.

Subjects: economy and rural development; farming systems; vegetal productions.

a culture cotonnière fut promue au Cameroun, en Centrafrique et au Tchad par l'administration coloniale dans les années 1940. Mais ce n'est que dans les années 1980 que la production de coton augmenta rapidement au Cameroun et au Tchad, quoiqu'à un degré moindre en Centrafrique. Cependant, depuis quelques années, les changements dans les conditions d'accès à la production cotonnière et l'évolution du commerce international du coton mettent les filières en difficulté, conduisent à leur réorganisation, et amènent en retour les producteurs à réduire la place du coton dans leurs stratégies de production.

Cet article présente l'évolution des politiques cotonnières en Afrique centrale, analyse leurs impacts à partir des résultats d'une dizaine d'années de recherche et présente des propositions d'accompagnement des acteurs de ces filières, en s'appuyant sur des études sur les filières cotonnières et les exploitations agricoles (EA) réalisées depuis 1999 dans les zones cotonnières d'Afrique centrale (figure 1).

\section{Évolution}

\section{des politiques cotonnières}

\section{dans un contexte} de crise

Depuis les années 1980, les politiques cotonnières évoluent dans le cadre d'un modèle économique ultralibéral. Ses tables de la loi, devenues célèbres sous le nom de "Consensus de Washington ", énumèrent les commandements auxquels devaient se plier les États : dérégulation, privatisation, monétarisme, réduction des dépenses publiques, etc. Cette charte libérale, adoptée par le Fonds monétaire international (FMI) et la Banque mondiale
(BM), a été à l'origine des politiques d'ajustements structurels (PAS) exigées des pays en développement en échange de la renégociation de leur dette. Les effets néfastes nés de l'application des règles du Consensus de Washington sont de plus en plus criants. Ces PAS sont qualifiées de "solutions archaïques et inadaptées, [décidées] sans tenir compte des effets qu'elles auraient sur les habitants des pays "(Stiglitz, 2003). La crise mondiale de la filière cotonnière a accentué ces effets en Afrique.

\section{Crises cotonnières et réformes en Afrique Centrale}

Les crises cotonnières africaines résultent des fluctuations et de la baisse tendancielle des cours internationaux de la fibre, de la faillite économique des États et du reflux des aides à l'agriculture (Gafsi et Mbétid-Bessane, 2003), mais aussi de facteurs structurels (dysfonction-

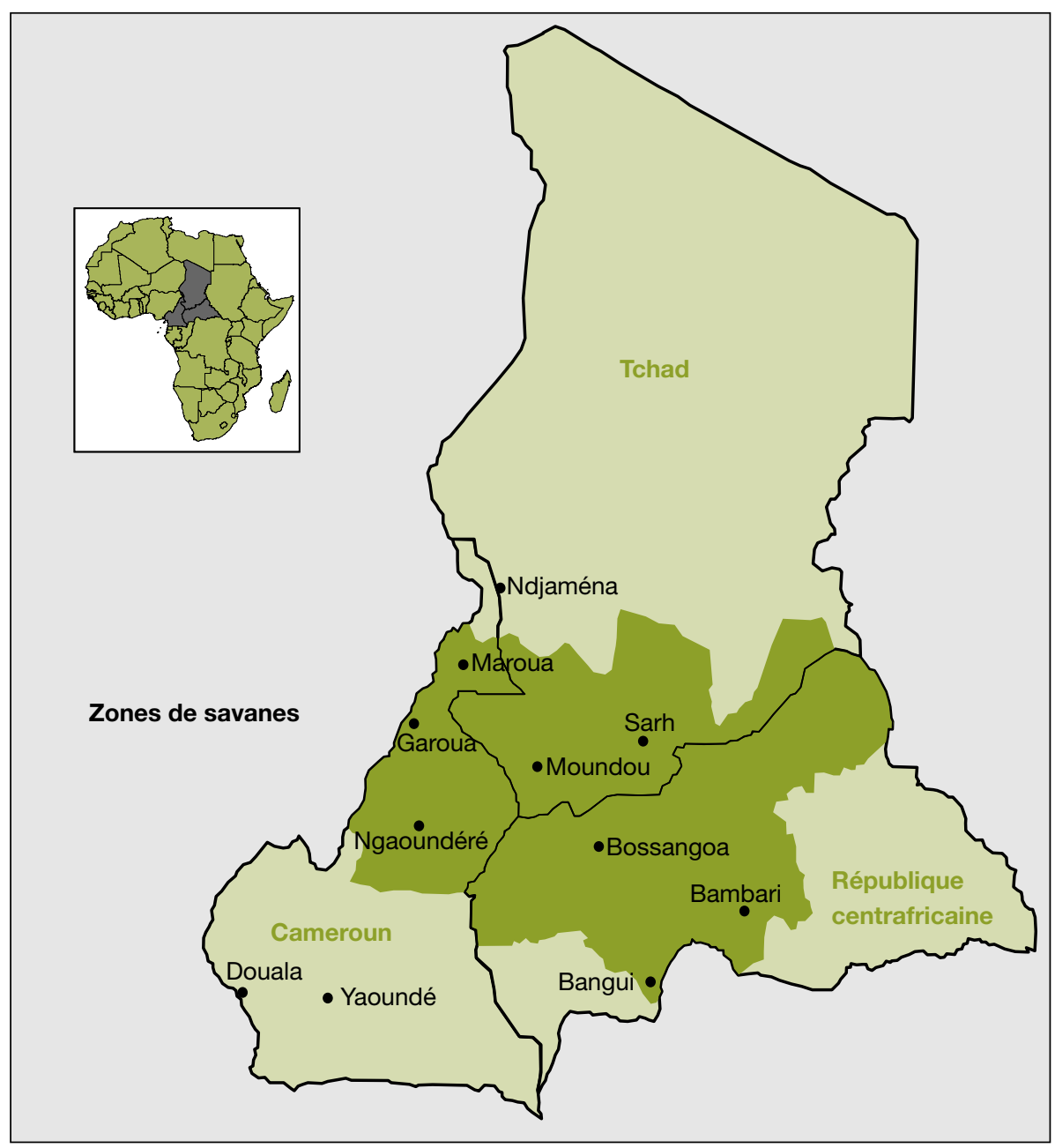

Figure 1. Carte de localisation des zones de savanes cotonnières en Centrafrique, Cameroun et Tchad.

Figure 1. Map of cotton production zones in Central African Republic, Cameroon and Chad savannahs. 
nement des filières, erreurs de gestion dans les sociétés cotonnières). L'agriculture africaine se trouve être la plus pauvre et la moins subventionnée du monde. La compétition est inégale avec les États-Unis et l'Europe, où les subventions déconnectent les exportations de coton des prix mondiaux. Avec $16 \%$ de contribution sur le marché mondial de la fibre, l'Afrique francophone ne peut que subir les fluctuations des prix mondiaux (Hugon, 1999).

Après la crise cotonnière de 1985-1986, les États et les sociétés cotonnières d'Afrique centrale ont procédé à des réformes prudentes : autonomisation des sociétés devenant des entreprises publiques à caractère industriel et commercial, recentrage sur la filière cotonnière, réduction des charges considérées comme improductives, etc. La dévaluation du franc CFA en 1994 a permis au coton de reprendre une place stratégique dans les économies nationales, de participer au développement du monde rural et d'assurer une part importante des revenus des populations des zones cotonnières (Berti et al., 2006). Ainsi, en 1998, l'Afrique se plaçait au troisième rang des exportateurs mondiaux, avec 950000 tonnes de fibre (Jouve, 1999). La BM considérait alors que les conditions pour la privatisation et le démantèlement du monopole des sociétés cotonnières étaient réunies, tandis que les partenaires franco-africains considéraient de leur côté que l'on pouvait faire évoluer l'organisation des filières en faisant participer les producteurs au capital des sociétés.
Le débat fit l'objet d'une controverse théorique entre les économistes autour de deux schémas de réorganisation de la filière : libéralisation complète ou intégration. Pour les adeptes de l'approche orthodoxe néoclassique, le marché était le seul mode de coordination efficient (Cahuc, 1993) : les filières devaient être démantelées, permettant la création de sociétés cotonnières en concurrence, pour améliorer leurs performances globales. Pour les adeptes des approches économiques hétérodoxes, les modes de coordination devaient prendre en compte l'institution, notamment l'État, l'organisation, les règles, les conventions, etc. (Valceschini, 1995 ; Ménard, 1997) : les filières intégrées par un seul opérateur devaient donc être maintenues.

Ce débat reste ouvert aujourd'hui en Afrique centrale, mais dans un contexte qui a fortement évolué : filières en déficit et en restructuration non achevée, promotion des organisations professionnelles, et, plus récemment, baisse du prix d'achat du coton et hausse des prix des intrants - excepté au Tchad (figure 2) et changements pour les producteurs des conditions de production et dans les transactions avec les sociétés cotonnières.

\section{Changements dans les conditions de production du coton}

Les baisses de la production cotonnière constatées depuis 2005 en Afrique centrale (tableau 1), qui se vérifient dans toute l'Afrique francophone (Perennes et
Esteulle, 2008), sont liées aux changements dans les conditions d'accès à la production et aux moyens de production.

En effet, les limites des zones cotonnières ont évolué différemment selon les pays. Au Cameroun, cette zone, localisée dans la région de l'Extrême-Nord dans les années 1950, s'est déplacée progressivement vers la région du Nord, peu peuplée et offrant de nouveaux espaces agricoles. Avec la crise actuelle, la Société de développement du coton au Cameroun (Sodecoton) envisage un nouveau mouvement géographique en recentrant la production sur les zones favorables : les niveaux de production y sont plus élevés et les usines plus proches. En Centrafrique, la zone cotonnière, jadis étendue à presque tout le territoire, a d'abord été réduite dans les années 1970, puis, après les crises cotonnières de 1985 et 1992, concentrée aux zones favorables. Mais, depuis 20052006, la chute de la production liée à l'insécurité et aux difficultés financières de la Société centrafricaine de développement des textiles (Socadetex) compromet l'avenir de la filière. Au Tchad, la Cotontchad a d'abord abandonné les régions du Nord et de l'Est après les crises cotonnières de 1985 et 1992, puis y a repris la production à la faveur du boom cotonnier à la fin des années 1990. La baisse actuelle des superficies, consécutive au déficit de la Cotontchad (cumul de 34 milliards de FCFA en 2008), peut avoir pour résultat, si elle se poursuit, de concentrer la production dans les zones favorables.

Les conditions d'accès aux moyens de production ont changé depuis le milieu des années 1980, plus spécifiquement en

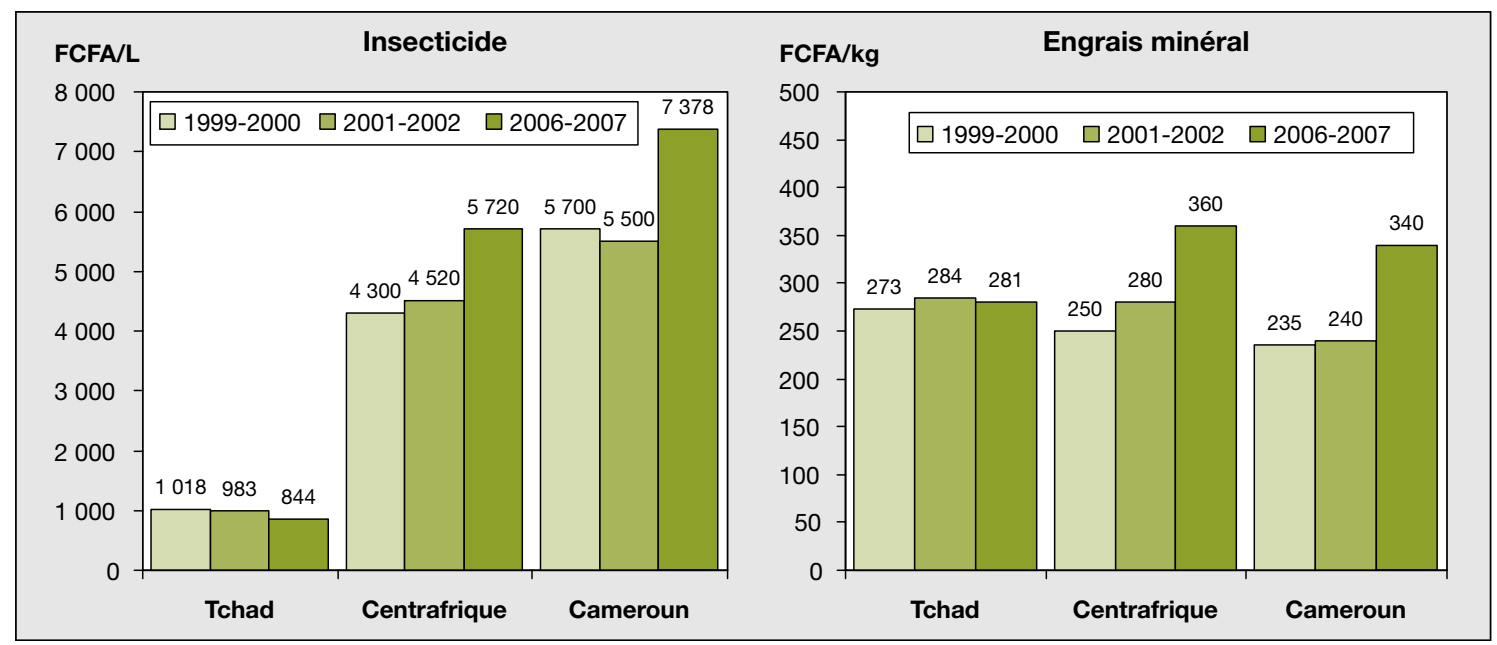

Figure 2. Évolution des prix des intrants de la culture cotonnière en Afrique centrale.

Figure 2. Evolution of cotton input prices in Central Africa. 
Tableau 1. Évolution de la production, des superficies et des rendements du coton en Afrique centrale.

Table 1. Evolution of cotton production, areas and yields in Central Africa.

\begin{tabular}{lrrrrrrr}
\hline Afrique centrale & $\mathbf{1 9 8 0}$ & $\mathbf{1 9 9 0}$ & $\mathbf{1 9 9 4}$ & $\mathbf{2 0 0 0}$ & $\mathbf{2 0 0 5}$ & $\mathbf{2 0 0 6}$ & $\mathbf{2 0 0 7}$ \\
& $\mathbf{1 9 8 1}$ & $\mathbf{1 9 9 1}$ & $\mathbf{1 9 9 5}$ & $\mathbf{2 0 0 1}$ & $\mathbf{2 0 0 6}$ & $\mathbf{2 0 0 7}$ & $\mathbf{2 0 0 8}$ \\
\hline Cameroun & & & & & & & \\
Surface (1 000 hectares) & 65 & 94 & 141 & 199 & 232 & 203 & 138 \\
Production* (1 000 tonnes) & 84 & 113 & 153 & 225 & 221 & 184 & 116 \\
Rendement (kg/hectare) & 1290 & 1200 & 1090 & 1130 & 950 & 905 & 844 \\
Centrafrique & & & & & & & \\
Surface (1 000 hectares) & 81 & 47 & 51 & 39 & 14 & 7 & 14 \\
Production* (1 000 tonnes) & 23 & 37 & 16 & 25 & 3 & 2 & 8 \\
Rendement (kg/hectare) & 280 & 790 & 310 & 640 & 272 & 363 & 570 \\
Tchad & & & & & & & \\
Surface (1 000 hectares) & 166 & 207 & 203 & 240 & 287 & 229 & 194 \\
Production* (1 000 tonnes) & 86 & 157 & 95 & 143 & 215 & 177 & 149 \\
Rendement (kg/hectare) & 520 & 760 & 470 & 600 & 748 & 774 & 767 \\
\hline
\end{tabular}

* Production en coton-graine.

Source : sociétés cotonnières d'Afrique Centrale.

termes de réduction des subventions sur les intrants et des montants des crédits sur les équipements agricoles. En réponse, les producteurs diminuent l'utilisation des intrants et le recours à la traction animale, ce qui entraîne une baisse des rendements du coton, mais aussi de la production des autres cultures bénéficiant, directement ou indirectement, des intrants du coton.

\section{Changements dans les conditions de transaction}

Ces changements portent sur les modifications dans la fixation des prix du cotongraine aux producteurs et les hausses des prix des intrants.

Le prix du coton-graine est passé d'un prix fixe, déterminé jusqu'en 1989 en début de campagne, à un prix minimum, fonction du prix sur le marché mondial, discuté avec les paysans (Fok, 2007). Avant 2002, un mécanisme de stabilisation permettait d'amortir les fluctuations du prix sur le marché mondial. À partir de 2002, le mécanisme tampon n'existe plus et les fluctuations des prix mondiaux sont directement répercutées aux producteurs, soit une tendance à la baisse depuis 2005 (tableau 2).

La baisse du prix d'achat du coton-graine, combinée à une augmentation des coûts des intrants, se traduit par une forte diminution de la valorisation économique du travail des paysans, représentée par une chute de la marge après remboursement des intrants (figure 3).

L'intérêt économique des producteurs pour le coton diminue par rapport au maïs, à l'arachide, etc. Pour encourager les producteurs à améliorer la qualité de leur coton-graine, le prix d'achat est accompagné de mesures incitatives. En Centrafrique, en 2008, trois prix d'achat sont pratiqués, selon trois niveaux de qualité (150 FCFA/kg, 105 FCFA $/ \mathrm{kg}$, 75 FCFA $/ \mathrm{kg}$ ), auxquels s'ajoutent la prime de précocité pour le coton-graine récolté et vendu entre décembre et janvier ( $5 \mathrm{FCFA} / \mathrm{kg}$ ) et les ristournes (4,27 FCFA) $\mathrm{kg}$ ). Au Cameroun, en 2008, trois prix sont pratiqués, selon la qualité (185 FCFA $/ \mathrm{kg}$, $175 \mathrm{FCFA} / \mathrm{kg}, 160 \mathrm{FCFA} / \mathrm{kg}$ ), et une prime de production (15 FCFA/kg) si le seuil de 200000 tonnes est dépassé. Mais les résultats escomptés n'ont pas été atteints, car les producteurs estiment que ces mesures incitatives restent en deçà des efforts consentis pour améliorer la qualité du coton-graine.

Les difficultés financières des sociétés cotonnières entraînent des retards dans le paiement du coton-graine aux producteurs. Ces retards, pouvant atteindre plusieurs mois, perturbent le plan de trésorerie et la réalisation des investissements des producteurs, et ils occasionnent des baisses des revenus tirés du coton.

Ces changements accroissent les risques et les incertitudes des producteurs, augmentent leur mécontentement et leur méfiance vis-à-vis des sociétés cotonnières et influencent négativement leur motivation à produire du coton.

\section{Impacts des politiques et enseignements à tirer}

\section{Adhésion perturbée des paysans à la production cotonnière}

Au début des années 2000, l'exploitation agricole moyenne cultivait entre 2,2 hectares (Centrafrique et Cameroun) et 2,8 hectares (Tchad), pour un revenu

\section{Tableau 2. Prix moyen d'achat du coton-graine au producteur en Afrique centrale.}

Table 2. Average purchase price of seed cotton in Central Africa.

\begin{tabular}{|c|c|c|c|c|c|c|c|c|c|}
\hline $\begin{array}{l}\text { Afrique } \\
\text { centrale }\end{array}$ & $1999-2000$ & $2000-2001$ & 2001-2002 & $2002-2003$ & $2003-2004$ & 2004-2005 & $2005-2006$ & 2006-2007 & 2007-2008 \\
\hline Cameroun & 175 & 185 & 180 & 175 & 185 & 185 & 170 & 175 & 185 \\
\hline Centrafrique & 150 & 150 & 150 & 150 & 150 & 150 & 150 & 159 & 150 \\
\hline Tchad & 170 & 150 & 165 & 170 & 160 & 175 & 160 & 160 & 160 \\
\hline
\end{tabular}

Prix moyens d'achat pour la qualité 1 (le prix payé au producteur prend de plus en compte d'éventuelles ristournes et mesures incitatives). Source: sociétés cotonnières d'Afrique centrale. 


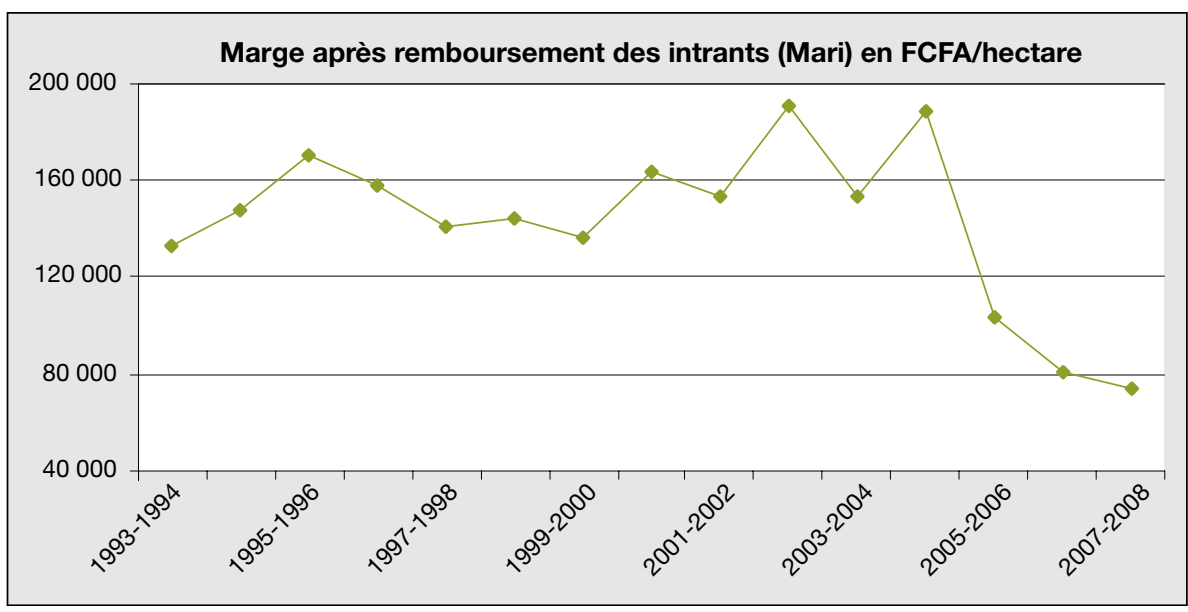

Figure 3.Évolution de la marge après remboursement des intrants en culture cotonnière au NordCameroun entre 1993 et 2008.

Figure 3. Evolution of cotton gross margin after repayment of production inputs in North Cameroon between 1993 and 2008.

annuel moyen de 220000 FCFA au Tchad, 250000 FCFA en Centrafrique et 270000 FCFA au Cameroun (MbétidBessane et al., 2006). Des coûts d'intrants élevés et des prix du coton à la baisse sont à l'origine du recul du coton dans les exploitations agricoles entre 2005 et 2008 (figure 4).

La répartition des trois types de stratégies cotonnières des producteurs identifiés en 1997-2000 a elle aussi évolué entre 2005 et 2008 (figure 5).

En effet, les producteurs (environ 1/3) ayant abandonné le coton entre 2005 et 2008 sont essentiellement ceux des types " croient au coton " et " culture de diversi- fication ". Ceux du type "opportunistes " produisent du coton quand le prix est à la hausse mais en réduisent fortement les superficies, voire l'abandonnent, quand le prix baisse, espérant le cultiver à nouveau quand les conditions de production redeviendront incitatives. Pour remplacer la culture et les revenus du coton, certains producteurs mettent l'accent sur les autres cultures (manioc et arachide en Centrafrique, maraîchage et arachide au Cameroun), d'autres développent l'élevage (bovins, petits ruminants et porcs, surtout) et/ou des activités extra-agricoles (chasse, pêche, cueillette, apiculture en Centrafrique, artisanat, bière de mil, petit commerce, manœuvre au Cameroun et au Tchad). De leur côté, les effectifs des producteurs qui " croient au coton " diminuent surtout au Cameroun dans les zones où existent des opportunités de diversification liées aux marchés urbains, mais aussi en Centrafrique, à cause de l'insécurité dans la zone de production. Ces producteurs sont à la tête de "grandes" exploitations agricoles dégageant des revenus monétaires élevés assurés aux 2/3 par le coton, et investissant dans l'élevage, la traction animale, le foncier, et dans des biens non productifs : maisons. Ils développent des stratégies d'augmentation des surfaces en coton et de commercialisation des vivriers pour maintenir leurs revenus. Les producteurs du type "culture de diversification", moins dépendants du coton que ceux qui "croient au coton", sont les plus nombreux à avoir abandonné le coton au profit d'activités variées.

Globalement, les exploitations agricoles montrent une réelle capacité d'adaptation au changement, mais, malheureusement, toutes ne se trouvent pas dans un environnement favorable à la diversification des activités - situation qui pourrait changer si le réseau des communications (routes et pistes) était amélioré.

\section{Inquiétudes sur le maintien des filières cotonnières et des services liés}

En Afrique centrale, la culture du coton mobilise plus de 500000 producteurs recevant quelques dizaines de milliards

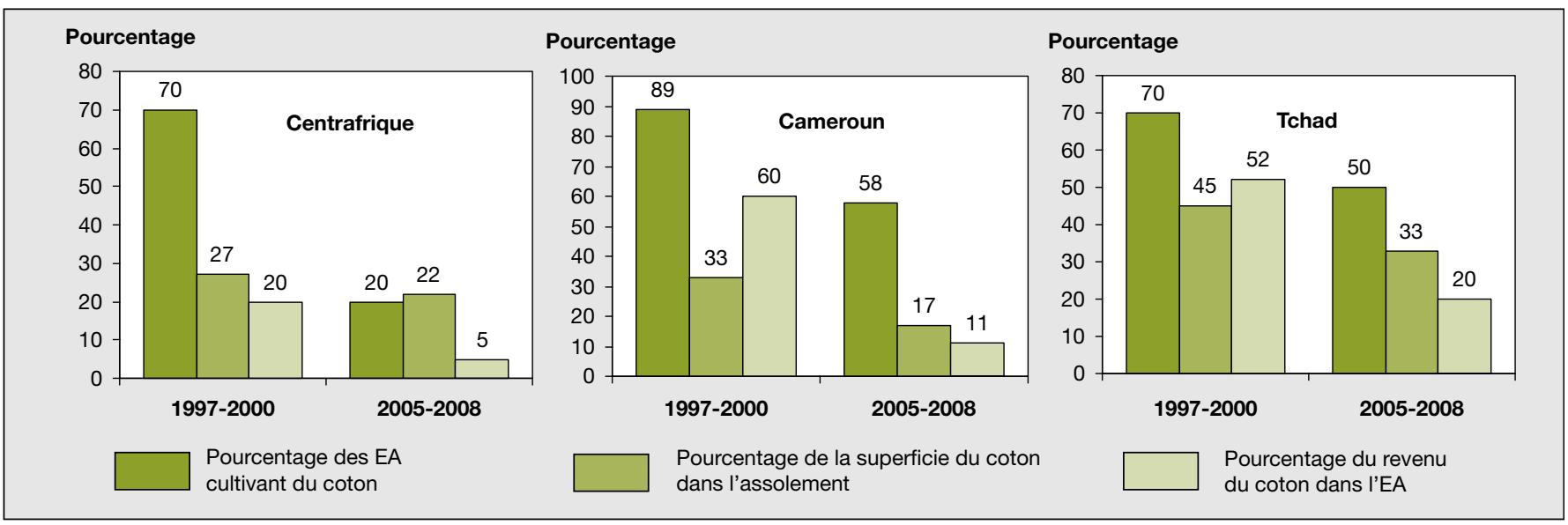

Figure 4. Évolution de la place du coton dans les exploitations agricoles enquêtées entre 1997-2000 et 2005-2008.

Figure 4. Evolution of the cotton area shares in farms investigated between 1997-2000 and 2005-2008. 


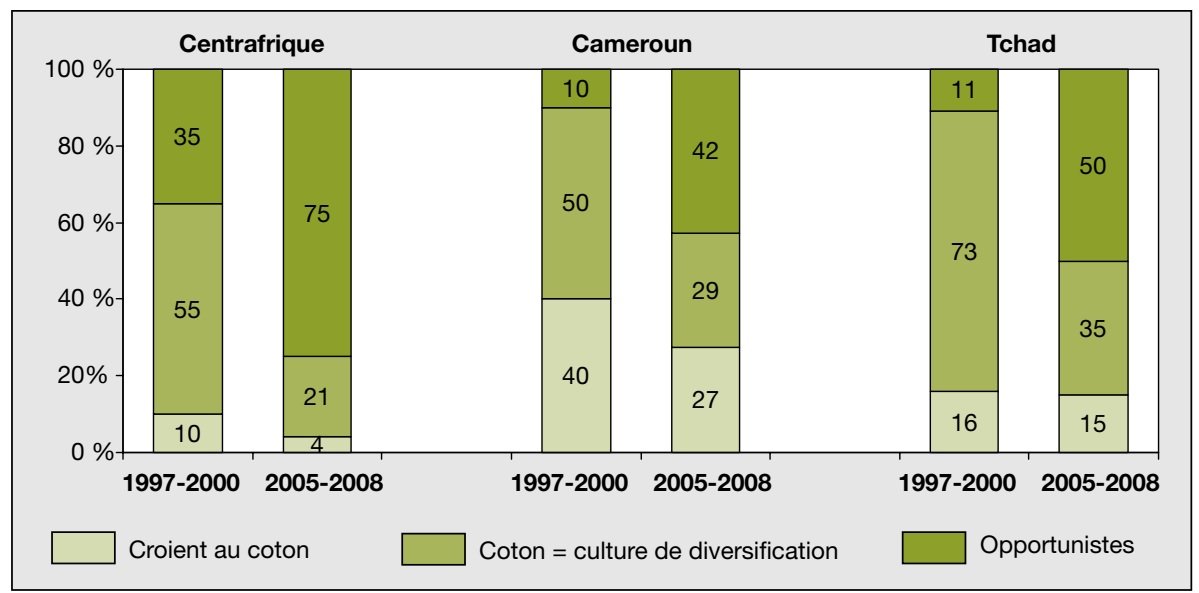

Figure 5. Indications des stratégies adaptatives des producteurs de coton en Afrique centrale.

Figure 5. Indications about cotton producers' adjustment strategies in Central Africa.

de FCFA de revenus annuels. Les graines de coton et leurs sous-produits sont généralement consommés localement - huile alimentaire, tourteaux, savon... - et la fibre est utilisée dans l'industrie textile (Estur, 2006). La vente du coton-fibre contribue significativement à la valeur des exportations agricoles. Au Cameroun, le coton a contribué, en 2004, à 76,7 milliards de FCFA, soit 5,2\% des recettes d'exportations - en position "franco à bord "-, et, en 2007, à 50,9 milliards de FCFA, soit 2,6\% (Folefack et al., 2009). Cette vente permet aux États de percevoir plusieurs milliards d'impôts et taxes chaque année. La production, la commercialisation et la transformation du coton créent plusieurs milliers d'emplois, directs (usines d'égrenage, huilerie, dispositif d'encadrement) et indirects (commerces, restaurants, mécaniciens, etc.). Les recettes de la production cotonnière permettent aussi d'assurer l'entretien de milliers de kilomètres de pistes rurales et le financement d'infrastructures sociales dans les villages. Enfin, la production du coton a favorisé le développement de fabricants de matériels agricoles, de transporteurs privés, d'organisations de producteurs (OP), etc. Le développement des OP est spectaculaire, mais leur pérennité n'est pas assurée, car de nombreux groupements de producteurs (GP) de coton présentent des dysfonctionnements caractéristiques, sont peu autonomes et manquent d'une assise professionnelle affirmée (Kossoumna Liba'a et Havard, 2006).

Les fortes chutes de production de ces dernières années (tableau 1) se traduisent par une baisse des revenus cotonniers des producteurs, de l'activité industrielle, entraînant une remise en cause de la rentabilité des usines et des services liés au coton. Si la crise perdure, les filières cotonnières, le tissu industriel et les services liés pourraient disparaître en Afrique centrale.

\section{Conclusion}

En Afrique centrale, les résultats des politiques cotonnières n'ont pas répondu aux attentes: peu de privatisations ont eu lieu; les crises des filières cotonnières entraînent la chute de la production ; les relations entre les producteurs, les OP et les sociétés cotonnières se détériorent; les GP et les OP dont l'endettement augmente sont fragilisés. Les sociétés cotonnières déficitaires réduisent leurs services aux producteurs, demandent des subventions aux États, cherchent à améliorer la rentabilité et à diversifier leurs activités.

Les changements de politique et la crise cotonnière affectent davantage le Cameroun et le Tchad. Ils posent les questions du maintien des sociétés cotonnières et de leurs infrastructures, mais aussi du développement des zones de savanes, supporté surtout par le coton ces dernières décennies. Le maintien d'un tissu industriel et des revenus des populations suppose d'améliorer les performances techniques et économiques des filières cotonnières, de revoir les conditions d'accès à la production de coton - avec certainement une implication forte des États, voire de la communauté internationale.
Dans un tel contexte, il est important d'accompagner les producteurs dans leurs stratégies d'adaptation aux changements. Ceci demande de faire évoluer les dispositifs d'appui, conçus pour la diffusion de messages techniques, vers le renforcement des capacités de ces producteurs (formation et conseil). Pour ce faire, le Pôle de recherche appliquée au développement des systèmes agricoles d'Afrique centrale (Prasac), a développé le conseil à l'exploitation familiale (CEF) (Djamen Nana et al., 2003). Depuis 2004, la Sodecoton a repris à son compte des activités de CEF à l'intention des GP (MbétidBessane et Havard, 2009) ; elle prévoit de l'étendre à un nombre croissant de GP dans les années à venir.

De même, des alternatives de développement de ces zones cotonnières doivent être envisagées : diversification agricole et développement de l'élevage en réponse aux besoins des marchés urbains, développement du tourisme et rétribution des services environnementaux.

\section{Références}

Berti F, Hofs JL, Sery Zagbaï H, Lebailly P. Le coton dans le monde, place du coton africain et principaux enjeux. Biotechnol Agron Soc Environ 2006 ; 10 : 271-80.

Cahuc P. La nouvelle micro-économie. Collection Repères. Paris: La Découverte, 1993.

Djamen Nana $\mathrm{P}$, Djonnéwa A, Havard M, Legile A. Former et conseiller les agriculteurs du Nord-Cameroun pour renforcer leurs capacités de prise de décision. Cah Agric 2003; 12: 241-5.

Estur G. Le marché mondial du coton : évolution et perspectives. Cah Agric 2006; 15 : 9-16.

Fok M. Rôles de l'État dans la construction et la restructuration des filières cotonnières en Afrique : Analyse par la théorie des réseaux. XXIII ${ }^{\mathrm{e}}$ Journées du Développement de I'Association Tiers-Monde "L'Etat malgré tout? Acteurs publics et développement ", Mons, 2007.

Folefack DP, Klassou C, Enam J. Price Adjustements to cotton crisis in Cameroon: Factors and consequences of farmers' adaptations. Life Sciences International Journal 2009 ; Special Issue N॰1 "Cotton Policies" : 89-99.

Gafsi M, Mbétid-Bessane E. Stratégies des exploitations cotonnières et libéralisation de la filière. Cah Agric 2003 ; 12 : 253-60.

Hugon P. Le "consensus de Washington " en question. Revue Tiers Monde 1999; 157: 11-36.

Jouve E. Une filière qui a fait ses preuves Problèmes économiques 1999 ; 2621 : 27-30.

Kossoumna Liba'a N, Havard M. Mutations de la filière cotonnière dans les provinces septentrionales du Cameroun. Perception et stratégies paysannes. Cahiers de Géographie du Québec 2006 ; 50 : 65-82. 
Mbétid-Bessane $E$, Havard M, Djondang $K$. Evolution des pratiques de gestion dans les exploitations agricoles familiales des savanes cotonnières d'Afrique centrale. Cah Agric $2006 ; 15: 555-61$.

Mbétid-Bessane E, Havard M. Cotton Policies in Central Africa and Farmers' Adaptive Strategies: How to assist them this process ? Life Sciences International Journal 2009, Special Issue N`1 "Cotton Policies": 100-11.
Ménard C. L'économie des organisations. Collection Repères. Paris: La Découverte, 1997.

Perennes J, Esteulle B. Production cotonnière africaine - campagne 2006-2007. Note de conjoncture $\mathrm{n}^{\circ} 16$. Paris: Service communication et relation publiques de la Présidence de Dagris, 2008.
Sitglitz J. La grande désillusion. Paris: Plon; Livre de Poche, 2003;

Valceschini E. Contrat, coordination et institutions. In: Allaire G, Boyer R, eds. La grande transformation de l'agriculture. Paris: InraEconomica, 1995. 